\title{
Manejo integral de la incontinencia urinaria femenina
}

\author{
Herman Redondo*; Raúl Cruz Palacio**; Francisco Sales P.***
}

\begin{abstract}
RESUMEN: Se realizó un trabajo prospectivo de ocho años de seguimiento, a partir del $1^{\circ}$ de agosto de 1983 , a las usuarias de la Clínica de Incontinencia Urinaria Femenina del Hospital Regional Simón Bolívar de Santafé de Bogotá, D.C.

Un total de 530 pacientes fueron estudiadas. Se presenta un protocolo de estudio clínico y un enfoque terapéutico, el cual divide las pacientes en siete grupos. La recaída global del trabajo realizado fue del $4.5 \%$, lo cual es satisfactorio y nos permite recomendar su utilización en otras instituciones.
\end{abstract}

PALABRAS CLAVES: Incontinencia urinaria femenina.

SUMMARY: Begining $1^{\circ}$ august 1983 , and 8 years prospective study was initiated to evaluate the female urinary incontinence. In the Simon Bolivar's Hospital of Santafe de Bogota, D.C.

A total off 530 patients were studied. We present of clinical study and a therapeutic protocol, clasifying the patients in 7 groups wich cover all possibilities. The total recidive in our work was $4.5 \%$, wich is very satisfactory and acceptable, we sugest to extend this protocol to other institutions.

KEY WORDS: Female urinary incontinence.

\section{Introducción}

En la década del 70 se produce a nivel mundial una avalancha de literatura científica encaminada a introducir nuevos conceptos sobre la micción normal, los mecanismos normales de continencia vesical, el estudio dinámico de la función vesical y la fisiopatología de la incontinencia urinaria en la mujer, lo cual llevó a revaluar el tratamiento de este trastorno $(1,3,14-15,19-20)$.

El uso indiscriminado de la técnica descrita por Kelly (4), o cualquiera de sus modificaciones (12), condujo a una alta tasa de fracasos, razón por la cual un número superior a 100 métodos operatorios han sido descritos, sin que exista hasta el momento un método ideal para garantizar éxito terapéutico en todos los casos, más bien la tendencia es recurrir a varias modalidades o combinación de cirugías para obtener los mejores rendimientos $(1,5,8,11,13,17$ 18, 29).

\section{Definición}

De acuerdo con la definición de la Sociedad Internacional de Continencia, entendemos como tal, la pérdida

* Profesor Asistente Departamento de Ginecología y Obstetricia Escuela Colombiana de Medicina. Hospital Simón Bolívar, Santafé de Bogotá.

** Profesor Asistente. Servicio de Urología. Escuela Colombiana de Medicina. Hospital Simón Bolívar.

*** Residente de IV año de Ginecología y Obstetricia. Escuela Colombiana de Medicina. involuntaria de orina por la región genital lo cual ocasiona problemas sociales o de higiene, objetivamente demostrados. En nuestro medio la salida involuntaria de orina es muy común, particularmente en mujeres multíparas y de edad avanzada; la cual se manifiesta clínicamente desde la pérdida involuntaria de orina con grandes esfuerzos, el flujo espontáneo con esfuerzos leves, hasta la incontinencia desencadenada durante la urgencia por estímulo no relacionados con el esfuerzo, como sucede cuando existe inestabilidad del músculo detrusor de la vejiga (25).

\section{Incontinencia urinaria de esfuerzo}

Se presenta incontinencia urinaria de esfuerzo verdadera cuando se pierde orina involuntariamente con los aumentos bruscos de la presión intra abdominal. En este caso la presión abdominal se desplaza a la vejiga y la presión intravesical excede la presión uretral máxima, se vence la resistencia uretral y se produce la emisión involuntaria de orina. La incontinencia urinaria de esfuerzo puede presentarse al toser, reír, estornudar, correr, saltar, levantar objetos pesados o durante cualquier ejercicio que aumente bruscamente la presión abdominal (25).

Tanagho afirma que para una normal continencia vesical en la mujer, la uretra posterior debe tener localización intra abdominal. Cuando la uretra se encuentra en posición normal, el aumento de presión abdominal no vence la resistencia uretral, este hecho anatómico de la localización de la uretra y el cuello vesical en la zona de presión del abdomen, es la base de la mayor parte de los procedimien- 
tos quirúrgicos para corregir la incontinencia urinaria de esfuerzo (23).

En el presente estudio consideramos de gran importancia para corregir la incontinencia urinaria de esfuerzo los siguientes factores: (20).

a. El anclaje o soporte uretral tanto proximal como distal.

b. Restituir la posición anatómica del cuello vesical.

c. Controlar la movilidad del cuello vesical.

d. Evitar la disposición en forma de embudo del cuello vesical, lo cual facilita el gradiente de presión en el sentido de la uretra.

e. Aumentar la longitud funcional y la resistencia uretral.

f. El piso pélvico; se debe fortalecer el mecanismo uretral estriado extrínseco.

Cuando el anclaje o soporte uretral se pierde, disminuye la longitud eficaz de la uretra, se presenta hipermovilidad del cuello vesical y de la uretra, la presión abdominal se canaliza hacia el cuello dispuesto en embudo, lo cual hace imposible mantener la presión de cierre uretral (25). Es decir, para recuperar la continencia perdida en las mujeres, con incontinencia urinaria de esfuerzo es necesario rescatar los mecanismos no funcionantes y restituirlos a su función normal. En nuestro concepto sólo es posible lograr estos objetivos al practicar una uretrocistopexia, sea por vía abdominal, vaginal o combinada y complementarla con una perineorrafia para reforzar el diafragma urogenital.

\section{Inestabilidad del músculo detrusor}

Es la pérdida involuntaria de orina a través de la uretra íntegra durante el síntoma urgencia, descrita como un problema secundario a la presencia de contracciones patológicas no inhibidas del músculo detrusor de la vejiga, sin incontinencia de esfuerzo y con una vejiga que puede o no estar en posición anatómica. Desde el punto de vista clínico se caracteriza por la triada de frecuencia, urgencia e incontinencia de urgencia y desde el punto de vista urodinámico por la presencia de contracciones no inhibidas del detrusor superiores a $15 \mathrm{~cm}$ de agua, durante la fase de llenado vesical (2).

El tratamiento de estas pacientes debe basarse en el uso de anticolinérgicos y el entrenamiento vesical, el trastorno funcional puro no responde a cirugía $(2,7,9)$.

\section{Incontinencia urinaria mixta}

Una mujer puede presentar simultáneamente inestabilidad del detrusor, incontinencia de urgencia y quejarse de pérdida de orina con los esfuerzos. En ellas, lo primordial es tratar de establecer cual es la primaria o cual de los dos componentes predomina.

Los estudios de Mcguire demuestran que en pacientes con incontinencia de. urgencia evidente, frecuencia diurna y nocturna, asociada a inestabilidad del detrusor, si la incontinencia de esfuerzo está presente, el $85 \%$ de los casos mejoran con una operación apropiada (9). Sin embargo deberá tenerse presente, previo a la cirugía, como primer interés terapéutico el tratamiento médico completo del componente de inestabilidad.

En el presente estudio, antes de afrontar tratamiento operatorio de una mujer con incontinencia urinaria, le realizamos un estudio clínico enfocado a determinar cuál o cuáles de los mecanismos de continencia están fallando, para saber si se trata de un caso de incontinencia urinaria de esfuerzo "pura", o de una incontinencia urinaria por inestabilidad vesical, o si se trata de una combinación de las dos patologías. Así mismo, evaluamos si la paciente presenta o no, prolapso genital asociado a la continencia urinaria, en cuyo caso afrontamos con una técnica por vía vaginal para corregir por la misma, tanto el prolapso como la incontinencia, tal como lo recomienda Powel (13). Cuando no existe prolapso asociado preferimos la suspensión de uretra y vejiga por vía abdominal, tomando como soporte el ligamento de Cooper, tal como lo describe Tanagho (24).

Propendemos entonces, no recurrir a una sola técnica para todos los casos, si no más bien disponer de un arsenal terapéutico de varios procedimientos quirúrgicos, seleccionados con base en indicaciones por nosotros establecidas, para brindar a cada persona el procedimiento óptimo a su cuadro clínico.

Pretendemos al presentar este trabajo aportar un protocolo multidisciplinario de manejo integral de la mujer con incontinencia urinaria adaptado a las necesidades y limitaciones propias de nuestro medio y el cual pueda ser aplicado en otras instituciones.

\section{Material y métodos}

Se realizó un estudio prospectivo de ocho años de seguimiento a las usuarias de la Clínica de Incontinencia Urinaria del Hospital Regional Simón Bolívar de Santafé de Bogotá, D.C., comprendido entre agosto $1^{\circ}$ de 1983 y junio 30 de 1991.

No fueron incluidas en el estudio las pacientes con diagnóstico de incontinencia urinaria Grado I, o de grandes esfuerzos, a las cuales sólo se les ordenó ejercicios vesicales isométricos y observación. Igualmente a los casos con infección urinaria comprobada, se le formuló tratamiento específico y posteriormente con urocultivo de control negativo, fueron nuevamente evaluadas (15).

\section{Protocolo de estudio}

Todas las pacientes estudiadas fueron sometidas al siguiente protocolo de estudio clínico:

a. La paciente es remitida a la Clínica de Incontinencia por los Servicios de Urología o Ginecología, donde es evaluada inicialmente y se le solicitan los exámenes de rutina, incluido el urocultivo.

b.En la Clínica de Incontinencia se le realiza la anamnésis específica, examen gineco urológico completo, incluidas las diferentes pruebas de continencia, como son:

- Prueba de Bonney, modificada e invertida.

- Prueba de D'bain y de Marshell.

- Prueba de Q. tip.

- Prueba de tos forzada, tanto acostada como de pie y con más de $200 \mathrm{cc}$. de orina en vejiga.

- Pruebas de sensibilidad.

- Pruebas de provocación de contracción involuntaria del detrusor.

- Medición del residuo vesical.

- Medición y calibración de la uretra. 


\section{c. Uretrocistoscopia}

d. Se practica estudio urodinámico de acuerdo con las siguientes indicaciones:

- Recaída previa de una cirugía anterior.

- Frecuencia, nicturia, urgencia e incontinencia de urgencia.

- Enuresis frecuente.

- Falla manifiesta en el control voluntario de la micción.

- Pacientes seniles o psiquiátricas.

- Nulípara o adolescente con incontinencia de urgencia.

- Antecedentes de diabetes clínica.

- Antecedentes de radioterapia en zona pélvica.

- Hallazgos en la uretrocistoscopia que lo indiquen, como capacidad vesical disminuida (menor de 300 c.c.) o aumentada (mayor de 800 c.c.).

\section{Protocolo terapéutico}

Una vez establecido el diagnóstico del tipo de incontinencia urinaria, cuál o cuáles de los mecanismos de continencia están fallando, las pacientes se distribuyeron en siete grupos terapéuticos, a saber:

\section{Grupo I:}

Pacientes con diagnóstico de incontinencia urinaria "pura", sin prolapso (máximo pueden presentar cistocele I o cistocele II de predominio anterior).

Tratamiento:

Uretrocistopexia suprapúbica con perineorrafia complementaria.

\section{Grupo II:}

Pacientes con diagnóstico de incontinencia urinaria "pura", con prolapso genital asociado (desde cistocele III en adelante).

Tratamiento:

Uretrocistopexia infrapúbica y la corrección simultánea de los celes (histerectomía vaginal, reparación de Manchester, enterocele, etc.).

\section{Grupo III:}

Recaída de uno de los grupos anteriores. Urodinamia: vejiga estable.

Tratamiento:

Cervicosuspensión bajo visión endoscópica.

\section{Grupo IV:}

Pacientes con diagnóstico confirmado por urodinamia de inestabilidad vesical, "pura", sin componente de esfuerzo y no asociada a prolapso.

Tratamiento:

Médico exclusivamente, comprende:

- Apoyo psicológico orientado a la motivación.

- Reeducación vesical

- Ejercicios vesicales isométricos.

- Dilatación uretral, si es necesaria.

- Tratamiento farmacológico, el cual comprende:

a. Anticolinérgicọs: Bromuro de propantelina.

b. Antiespasmódicos: Flavoxato, azopiridina. c. Inhibidores de la MAO: Clorhidrato de imipramina.

d. Inhibidores del calcio: Flunarizina.

e. Alfa adrenérgicos: Fenilpropanolamina, estrógenos conjugados.

f. Antisépticos urinarios: Nitrofurantoina, mandelato de metenamina, trimetropin-sulfa. etc.

\section{Grupo V:}

Pacientes con diagnóstico clínico y urodinámico de incontinencia urinaria mixta, es decir, presentan simultáneamente componente de inestabilidad y de esfuerzo. Sin prolapso genital asociado (máximo se permite cistocele I ó II anterior).

Tratamiento:

Médico inicial. Uretrocistopexia suprapúbica y perineorrafia.

\section{Grupo VI:}

Pacientes con diagnóstico clínico y urodinámico de incontinencia mixta, asociada a prolapso genital (cistocele II en adelante).

Tratamiento:

Médico inicial. Uretrocistopexia infrapúbica más la corrección de los celes.

\section{Grupo VII:}

Pacientes con diagnóstico confirmado por urodinamia de vejiga inestable, "pura", sin componente de esfuerzo y asociada a prolapso genital.

Tratamiento:

Médico inicial. Corrección quirúrgica de los celes.

\section{Resultados}

Un total de 530 casos fueron estudiados, los cuales se distribuyeron en los siete grupos terapéuticos así:

\section{Grupo I}

Se realizaron en este grupo 254 uretrocistopexias suprapúbicas. Es el grupo mayoritario, corresponde al $47.9 \%$ del total, distribuidos así:

- Uretrocistopexia suprapúbica simple: 46 casos $(18.1 \%)$.

- Uretrocistopexia suprapúbica y perineorrafia: 179 casos $(70.4 \%)$.

- Uretrocistopexia suprapúbica e histerectomía total abdominal: 20 casos (7.8\%).

- Uretrocistopexia suprapúbica e histerectomía abdominal ampliada: 4 casos (1.6\%).

- Uretrocistopexia suprapúbica y extirpación de quiste de ovario: 5 casos $(1.9 \%)$.

\section{Grupo II}

Se practicaron 126 uretrocistopexias infrapúbicas en este grupo. Corresponde al $23.7 \%$ del total, distribuidas así:

- Uretrocistopexia infrapúbica más colpoperineorrafia: 80 casos $(63.5 \%)$.

- Uretrocistopexia infrapúbica más histerectomía vaginal: 25 casos (19.8\%).

- Uretrocistopexia infrapúbica más reparación de Manchester Fothergill: 12 casos (9.5\%). 
- Uretrocistopexia infrapúbica más corrección de enterocele y/o desgarro III complicado: 9 casos (7.1\%).

\section{Grupo III}

En total se practicaron quince cérvico-suspensiones bajo visión endoscópica, corresponden al $2.8 \%$ del total.

\section{Grupo IV}

Corresponde al grupo de tratamiento médico exclusivo, en pacientes con diagnóstico confirmado por estudio urodinámico de inestabilidad vesical "pura". No hablamos de curación en este grupo, si no más bien de mejoría de sus síntomas. Se reportaron 29 pacientes con vejiga inestable pura, $5,5 \%$ del total de las cuales experimentaron mejoría con el tratamiento prescrito $23(79 \%)$, y hubo seis casos $(21 \%)$, que nunca presentaron mejoría.

\section{Grupo V}

Se presentaron 59 casos de incontinencia urinaria mixta, sin prolapso, equivalente al $11.1 \%$ del total. A todas se les suministró tratamiento médico inicial y posteriormente se les practicó uretrocistopexia suprapúbica.

\section{Grupo VI}

Se presentaron 35 casos de incontinencia urinaria mixta, asociada con prolapso genital, equivalente al $6.6 \%$ del total. A todas estas pacientes se les administró tratamiento médico inicial antes de practicarles una uretrocistopexia infrapúbica.

\section{Grupo VII}

Se presentaron 12 casos de inestabilidad vesical, sin componente de esfuerzo, asociado a prolapso genital, equivale al $2.3 \%$ del total de pacientes estudiadas. A todas se les suministró tratamiento médico inicial y luego se les practicó la corrección del prolapso respectivo, así:

- Colpoperineorrafia: 7 casos $(58.3 \%)$.

- Histerectomía vaginal: 3 casos (25\%).

- Corrección de enterocele por vía vaginal: 2 casos $(16.6 \%)$.

\section{Resultados globales de todos los grupos Morbilidad global}

En la Tabla 7 se anota el informe de la morbilidad global de los 501 casos operados, donde se aprecia que la alguria, la retención e infección urinaria, así como el dolor pélvico, son las molestias más frecuentes en el estado post operatorio sin importar el tipo de cirugía; se excluyen los casos de tratamiento médico exclusivo (Grupo IV).

\section{Recaída global}

La recaída global de la incontinencia urinaria del protocolo de manejo integral propuesto, comparativa con todos los grupos, se reporta en el gráfico 2, y la tabla 8 precisa la recaída global correspondiente al $4.5 \%$. Para este cálculo se excluyeron las pacientes de manejo médico exclusivo (Grupo IV) y así mismo, las pacientes del grupo VII, por tratarse de cirugías de prolapso y no de incontinencia. El gráfico 3 muestra la aparición en el tiempo de dichas recaídas, donde se aprecia mayor concentración en los meses 6, 14 y 24.
Tabla 7

MORBILIDAD GENERAL DE TODOS LOS CASOS OPERADOS

\begin{tabular}{|l|c|c|}
\hline \multicolumn{1}{|c|}{ Morbilidad } & No. de casos & $\%$ \\
\hline Alguria & 76 & 15.5 \\
Retención urinaria & 71 & 14.1 \\
Infección urinaria & 58 & 11.5 \\
Dolor pélvico & 34 & 6.7 \\
Incontinencia de urgencia & 24 & 4.8 \\
Hematoma & 21 & 4.1 \\
Absceso & 11 & 2.2 \\
Fístula ves-cutánea & 2 & 0.4 \\
Osteitis pubis & 2 & 0.4 \\
Fístula ves-vaginal & 1 & 0.2 \\
Fístula recto-vaginal & 1 & 0.2 \\
& & \\
\hline Total casos & 501 & 100 \\
\hline
\end{tabular}

Tabla 8

DATOS RECAIDA GLOBAL DE TODAS LAS PACIENTES OPERADAS. CASOS OPERADOS PARA I.U.E.

\begin{tabular}{|c|c|c|}
\hline Total casos operados & Totalrecaídas & \% Global \\
\hline 489 & 22 & 4.5 \\
\hline
\end{tabular}

Total casos estudiados: 530 .

\section{Inestabilidad vesical global}

La sumatoria de los grupos IV al VII nos da el total de pacientes con inestabilidad vesical, son 135, de las cuales 106 fueron operadas, o sea el 78.5\% (ver Tabla 9).

Antes de proceder a operar una paciente con inestabilidad del detrusor y componente de esfuerzo, se tuvieron en cuenta unos criterios por nosotros establecidos.

\section{Criterios para operar pacientes con incontinencia urina-} ria mixta

a. La paciente debe entender y estar de acuerdo con el tratamiento.

b.En el estudio urodinámico, las contracciones no inhibidas del músculo detrusor serán de baja intensidad y preferiblemente aparecer al final de la fase de llenado.

c. Lo más importante: La respuesta clínica al tratamiento médico, el cual no se da por tiempo menor a un mes, antes de la eventual cirugía.

\section{Parto intervenido y macrosomía}

Hubo 31 casos de partos intervenidos como antecedente importante: 20 con fórceps y 11 con espátulas de Velasco. Así mismo, otro antecedente importante y estadísticamente 
Tabla 9

\section{ESTADISTICA DE PACIENTES CON INESTABILIDAD VESICAL TOTAL CASOS DE VEJIGA INESTABLE}

\begin{tabular}{|c|c|c|c|}
\hline Total casos & $\%$ & Operados & $\%$ \\
\hline 135 & 25.47 & 106 & 78.5 \\
\hline
\end{tabular}

Total casos estudiados: 530 .

significante, es la macrosomía, 76 de las 530 pacientes (el $14,3 \%$ ) lo presentaron.

Hallazgos en la Uretrocistoscopia:

- Calibre uretral promedio

22 a 24 FR

- Longitud uretral promedio

$3.56 \mathrm{~cm}$

- Capacidad vesical promedio

$435 \mathrm{cc}$

- Menor capacidad vesical

$200 \mathrm{cc}$

- Mayor capacidad vesical

$1000 \mathrm{cc}$

- Estrechez uretralel

$11.7 \%$

- Traveculación vesical

$4.2 \%$

$2.4 \%$

$0.9 \%$

- Divertículos intravesicales

$0.9 \%$

- Metaplasia escamosa del trígono

- Total cistoscopias realizadas 424 (al 80\% de las pacientes)

La uretrocistoscópia en el presente estudio es pilar fundamental para la valoración de la anatomía de la mujer incontinente. Preferimos un endoscopio de 12 o de 0 grados. Colocamos el aparato en la uretra distal o media y mantenemos el flujo de agua abierto y así determinamos el anclaje de la uretra proximal. A continuación se le solicita a la paciente aumento de presión abdominal mediante pujo para apreciar la movilidad del cuello y la uretra, posteriormente se penetra a vejiga y se observan otros aspectos morfológicos (19).

El control endoscópico post operatorio no lo practicamos de rutina, en las pacientes que lo hemos realizado, el resultado de los casos bien corregidos es siempre el de una uretra elongada, con buen soporte a nivel proximal como distal, de movilidad normal y posición anatómica del cuello intra abdominal. El residuo vesical promedio, un mes después de cirugía fue menor que la misma medida antes de cirugía, independiente de la vía escogida.

\section{Estudios urodinámicos}

Se practicaron 247 urodinamias, al $46 \%$ de la población estudiada, 135 casos fueron positivos para inestabilidad del músculo detrusor (el 54.6\%) distribuidos así:

Estudios practicados

Vejigas inestables

Casos de I.U. mixta

Casos de V.I. "pura"

Inestabilidad asociada a prolapso

Casos operados

\section{Morbilidad}

\section{Morbilidad selectiva del Grupo I}

En la morbilidad selectiva del grupo I es la retención urinaria y la alguria transitoria las molestias post operatorias más frecuentes. Se presentaron dos casos de fístula vesico cutánea, los cuales se corrigieron con drenaje vesical permanente durante quince días. Así mismo, hubo dos casos de hipercorrección los cuales requirieron reintervención un mes después. La recaída de este grupo es la más baja y corresponde al $2.36 \%$.

\section{Morbilidad selectiva del Grupo II}

En la morbilidad selectiva del grupo II se aprecia que la alguria transitoria y la retención urinaria son las molestias post-operatorias más frecuentes. Hubo dos casos de osteitis aséptica del pubis, los cuales recibieron tratamiento con piroxicam durante tres meses, al cabo de los cuales cedieron los síntomas. Se presentó un caso de fístula vesico vaginal postrigonal la cual se corrigió quirúrgicamente por vía vaginal, sin más complicaciones. La recaída en este grupo es superior al anterior y corresponde al $4.76 \%$.

\section{Morbilidad selectiva del Grupo III}

En la morbilidad de las pacientes del grupo III se observa que el dolor suprapúbico y la infección urinaria son las molestias post operatorias más frecuentes. La recaída por segunda oportunidad en este grupo fue de 4 casos, lo cual corresponde al $26.7 \%$.

\section{Morbilidad selectiva del Grupo V}

En la morbilidad selectiva de este grupo se observa que la alguria y la retención urinaria son nuevamente las molestias post operatorias más frecuentes. La recaída del componente de esfuerzo se presentó en tres casos, lo cual corresponde al $5.1 \%$ muy superior al anotado en el grupoI.

\section{Morbilidad selectiva del Grupo VI}

La morbilidad selectiva de este grupo muestra una recaída del componente de esfuerzo del $8.5 \%$, muy superior a la obtenida en el grupo II.

\section{Morbilidad selectiva del Grupo VII}

La morbilidad selectiva de este grupo fue muy baja. Hubo dos casos en los cuales persistió la inestabilidad vesical.

\section{Distribución de pacientes}

\section{Distribución de pacientes por grupo}

El gráfico 1 muestra como quedaron distribuidas las 530 pacientes estudiadas según los siete grupos terapéuticos propuestos, evidencia cómo los grupos I y II conforman el $70 \%$ de toda la población.

\section{Distribución de la recaída por grupos}

El gráfico 2 muestra la distribución de la recaída por grupos, en comparación con la recaída global. Se observa que la recaída en el grupo I, correspondiente a colpocistopexias por vía abdominal, es la menor, la cual se incrementa en el grupo $\mathrm{V}$ cuando se practica este tipo de 
Gráfico 1

DISTRIBUCION DE PACIENTES POR GRUPO

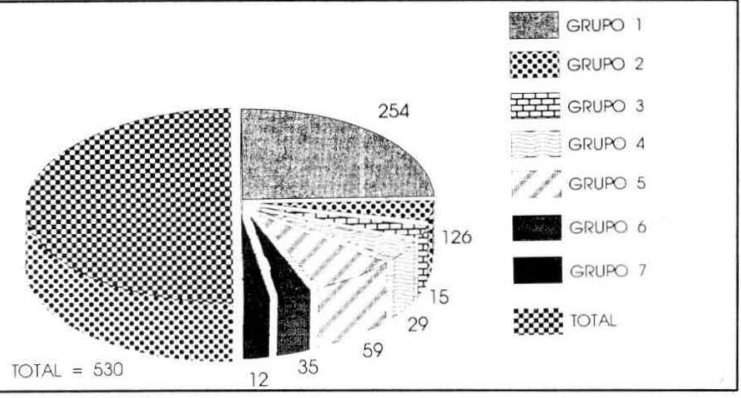

intervención a pacientes con incontinencia mixta. Así mismo, la técnica vaginal con incontinencia urinaria de esfuerzo "pura", da mejor rendimiento que cuando se utiliza en pacientes con incontinencia mixta, lo demuestra la comparación del grupo II (4.76\%), con la del grupo VI (8.57\%).

Gráfico 2

DISTRIBUCION DE RECAIDAS POR GRUPO
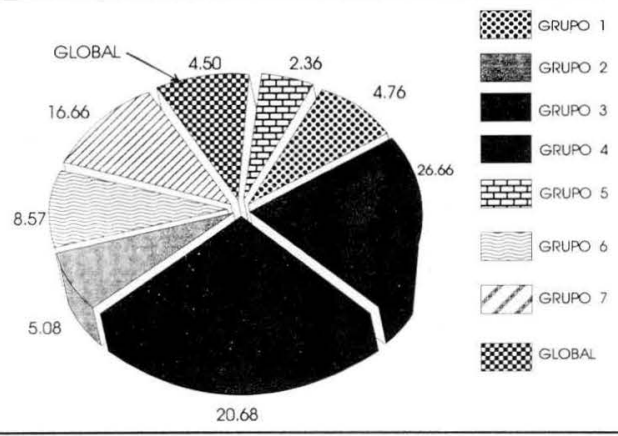

\section{Distribución de pacientes por edades}

La relación de la edad en el momento de iniciar el tratamiento, se encuentra distribuida en el Gráfico 4, donde se aprecia que la incontinencia urinaria aumenta notoriamente después de los 30 años y declina después de los 50.

Gráfico 3

\section{FRECUENCIA DE LAS RECAIDAS POR TIEMPO}

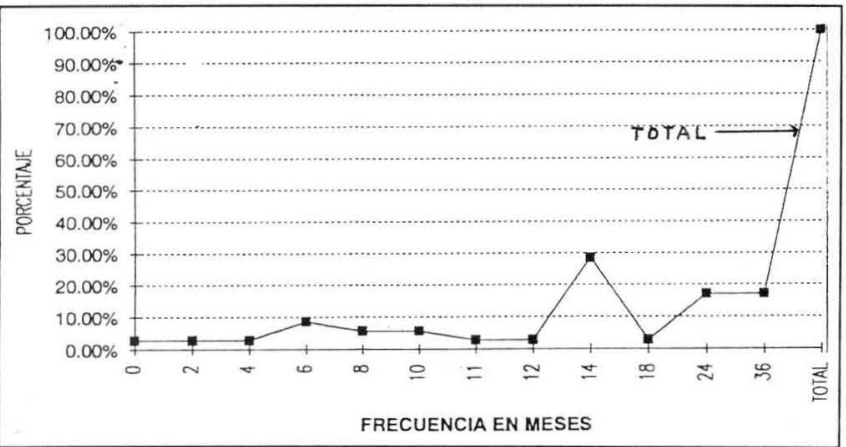

Si valoramos exclusivamente el caso de la inestabilidad vesical, observamos que declina más lentamente que la incontinencia urinaria de esfuerzo. La inestabilidad vesical es más frecuente en la tercera edad.

Gráfico 4

\section{DISTRIBUCION DE PACIENTES POR EDADES}

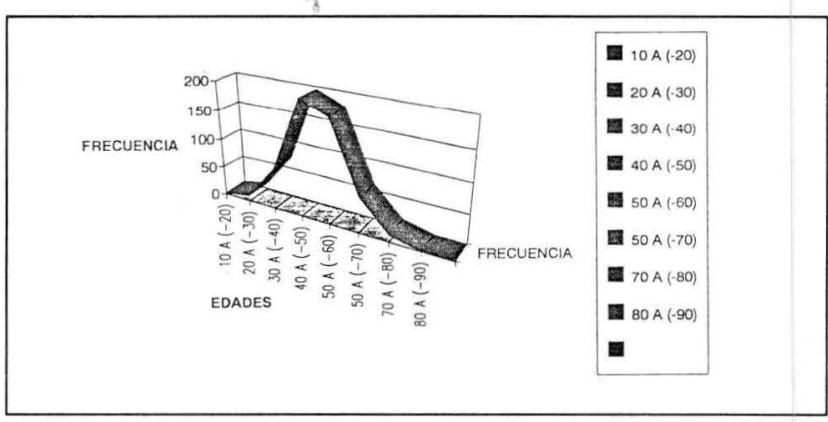

\section{Comentarios}

a. La cirugía más frecuentemente realizada fue la uretrocistopexia suprapúbica (313 casos, el 59\% del total), en 29 casos se aprovechó la misma vía para hacer otro tipo de cirugías, como histerectomía abdominal, total o ampliada, resección de quistes de ovarios o ligadura de trompas.

b. La segunda cirugía en frecuencia fue la uretrocistopexia infrapúbica, (161 casos, 30.3\% del total), de las cuales en todos los casos se aprovechó la misma vía para corregir los celes, tales como, colpoperineorrafias, histerectomías vaginales, corrección de enterocele o desgarro perineal.

c. A medida que se agudiza el estudio clínico nos damos cuenta que el porcentaje de las formas "puras", es menor. Particularmente los casos de V.I., casi siempre se acompañan de componente de esfuerzo. Cuando optamos por el tratamiento médico exclusivamente, hablamos sólo de mejoría en un $80 \%$ de los casos, también tenemos un porcentaje no cuantificado de deserciones al tratamiento en este grupo.

d. Observamos mayor tendencia al dolor suprapúbico en el caso de cirugía abdominal; en contraste esta vía tiene menor tendencia a la retención e infección urinaria o a recaídas.

e. La vía vaginal presenta mayor tendencia a la retención e infección urinaria, casi no deja secuela de dolor pélvico. Salvo en los dos casos reportados como osteitis aséptica del pubis, los cuales cedieron a los tres meses de tratamiento a base de anti inflamatorios.

f. El mayor porcentaje de recaídas se presentó al segundo y tercer año, resultado bien diferente al obtenido en nuestro primer reporte, en 1986, cuando observamos el mayor número de recaídas e el primer año (15). (Ver gráfico 3).

g. Tanto los casos de I.U.E., como de V.I., aumentaron con la edad, la V.I. aparece más temprano y declina un poco más tarde y se hace mucho más frecuente en la tercera edad. La forma pura de esfuerzo tiende a declinar después de la década de los 50.

h. La I.U. femenina en general aumenta progresivamente con el número de hijos. No observamos casos de I.U.E., en nulíparas y sí, 5 casos de V.I. 
i. Consideramos excesivo el número de estudios urodinámicos solicitados, el cual disminuirá en la medida en que se agudice el juicio clínico, sobre todo, si tenemos en cuenta el alto costo de este examen.

\section{BIBLIOGRAFIA}

1. Burch JC. Urethrovaginal fixation to Cooper's ligament for correction of stress incontinence, cystocele and prolapse. Am. J. Obstet. Gynecol. 1961; 81: 128.

2. Díaz J., Hernández C. Urodinamia en incontinencia urinaria femenina. Memorias primeras jornadas de ginecol. y obstet., Bogotá, 1984.

3. Hodkinson C.P. Stress urinary incontinence. Am. J. Obstet. and Gynecol. 1970; 108: 1141.

4. Kelly HA. Incontinence of urine in women. Urology and cutaneus review, 1913; 17, 291: 293.

5. Lomanto A., Gómez P. Operación de Kelly, Rev. Col. Obstet. y Ginecol. 1983; Vol. XXXIV, 28.

6. Lomanto A. Cols. Evaluación de la colpocistouretropexia en la incontinencia urinaria de esfuerzo. Rev. Col. Obstet. Ginecol. 1982; Vol. XXXIII, 112.

7. Lomanto A. Incontinencia urinaria de esfuerzo en la mujer, tratamiento. Primeras jornadas de ginecol. y obstet. Clínica Fray Bartolomé de las Casas, Memorias. Bogotá, 1982.

8. López LA., Corzo JE Corrección de la incontinencia urinaria de esfuerzo por medio de la uretrocistopexia retropúbica por vía vaginal. Rev. Col. Obstet. y Ginecol. 1983; Vol. XXXIV, 34.

9. McGuire EJ. Clinical evaluation of the female. Lower urinary tract. The urology clinics of N.A., 1985.

10. Marshall VF., Marchetti A., Krantz KE. The corrections of stress incontinence by simple vesicourethral suspension. Surgery Gynecol. and Obstet. 1949; 88: 509, 518.

11. Pereyra AJ., and Cols. Combined urethrovesical suspension and vaginourethroplasty of stress incontinence. Obstet. and Gynecol. 1976.

12. Pacey K. Patology and repair of genital prolapse. J. Obstet. Ginecol. Brith. 1949; 56.
13. Powell LC Jr. Rethropubic urethrocystopexy: Vaginal approach, Am. J. Obstet. and Ginecol. 1981; 140: 91.

14. Redondo H., Lomanto A. Revisión de la operación de Kelly en 10 años del IMI. Trabajo de promoción, inédito, Bogotá 1981.

15. Redondo H., Cruz R. Estudio clínico y enfoque terapéutico de la incontinencia urinaria en la mujer. Rev. Col. Obstet. y Ginecol. 1986; Vol. XXXVIII.

16. Redondo H. Técnicas vaginal para corregir I.U.E., Memorias. Curso de incontinencia urinaria, Instituto Materno Infantil, Bogotá. 1986.

17. Redondo H. Uretrocistopexia infrapúbica. Rev. Col. Obstet. y Ginecol. 1989; Vol. XXXIX.

18. Stamey TA. Surgery of female incontinence. Am. J. Obstet. y Ginecol. $1980 ; 77$.

19. Snyder Y., Lipsitz A. Evaluations of female urinary incontinence. The urology clinics of N.A., 1991.

20. Stuart L., Stanton P. Cirugía en la incontinencia urinaria, temas actuales de Ginecol. y Obstet. 1978; 5, 79.

21. Tanagho EA. Observations on the dinamics on the Bladdernck, Brith. J. Urol. 1966; 38, 72.

22. Tanagho EA. Mechanism as urinary continence embriogic, anatomic and pathologic considerations. Brit. J. Urol. 1968.

23. Tanagho EA. and Cols. The trigone: and physiologycal considerations in relations to the urethrovesical juntion. Am. J. Urol. 1968.

24. Tanagho EA. and Cols. Colpocystourethropexy: The way, we do it. Am. J. Urol., 1976; 110: 751.

25. Whithead D. Incontinence o urine in women. Mont Sinai, Brit. J. Urol. 1987. 